Ann. Zootech., I975, 24 (3), 35I-362.

\title{
INFLUENCE DE LA MACHINE DE RÉCOLTE SUR LA VALEUR DES ENSILAGES DE GRAMINÉES POUR LES GÉNISSES DE RACE LAITIERE
}

\author{
J. P. DULPHY et C. DEMARQUILLY \\ avec la collaboration technique de J.-P. ANDRIEU et J.-P. GAREL \\ Laboratoire des Aliments, \\ Centre de Recherches de Clermont-Ferrand, I. N.R. A., \\ Theix, Saint Genès Champanelle, 63110 Beaumont
}

\begin{abstract}
RÉSUMÉ
Au cours de 4 essais, nous avons comparé sur des génisses de race laitière des ensilages récoltés avec 3 machines différentes : ensileuse à tambour hacheur (brins courts), ensileuse à plateauhacheur (brins moyens), ensileuse ì fléaux (brins longs). Tous les fourrages ont été préparés avec addition d'acide formique à la récolte et le type de machine n'a eu qu'une influence faible sur la qualité de conservation des ensilages.

Ces ensilages ont été distribués ì volonté sans foin à des lots comparables de génisses de I an subdivisés en sons-lots recevant des quantités différentes d'aliment concentré ( $\mathrm{l}$ et $2 \mathrm{~kg}$ en moyenne).

Les quantités d'ensilage ingérées par les génisses ont été en moyenne plus élevées de 6,4 p. roo avec les ensilages à brins courts par rapport ì ccux à brins longs et sculement de 2,6 p. Ioo par rapport ì ceux à brins moyens. I.es augmentations correspondantes des gains de poids journalicrs ont ćté respectivement de +126 et +84 g/jour.

Lorsque nous avons fait varier lc niveau d'apport de l'aliment concentré nous avons enregistré une diminution moyenne de $0,43 \mathrm{~kg}$ de matière sèche d'ensilage par $\mathrm{kg}$ de matière sèche d'aliment concentré apporté en supplément et cela indépendamment de la finesse de hachage des ensilages. L'augmentation du gain de poids vif correspondante a été de $+86 \mathrm{~g} /$ jour et a été d'autant plus faible que le gain de poids des animaux ayant reçu le plus faible apport d'aliment concentré était élevé.

Les performances plus élevées des génisses avec les ensilages hachés finement semblent résulter pour une faible part de l'augmentation des quantités ingérées et pour une part plus importante d'une amélioration de l'efficacité d'utilisation de la matière organique digestible ingérée. İn revanche, l'augmentation de la quantité de matière organiçue digestible ingérée due à l'augmentation de l'apport d'aliment concentré semble surtout s'être traduite par une modification cle la composition du crôit.
\end{abstract}

\section{INTRODUCTION}

Le hachage fin des fourrages lors de la récolte permet d'améliorer considérablement leur qualité de conservation par ensilage (Dulphy et DEMARQUILLY, I973). Simultanément, il nous a permis de faire ingérer à des moutons des quantités de 
matière sèche élevées et bien supérieures $(+43,9$ p. IOo) à celles ingérées sous forme d'ensilage à brins longs. I a finesse de hachage est donc un facteur primordial pour améliorer l'ingestibilité des ensilages par le mouton; elle agit à la fois directement et par l'intermédiaire de la qualité de conservation. Nous avons donc voulu étudier s'il en était de même chez les bovins et, dans un premier temps, nous avons utilisé des génisses de I an.

A notre connaissance, une seule étude existe à ce sujet : celle de Roche, O'SHEA et FLYN en Irlande (I972). Ces auteurs ont trouvé un gain de poids supérieur (505 au lieu de $325 \mathrm{~g} /$ jour) pour des génisses đe 350 à $400 \mathrm{~kg}$ recevant de l'ensilage finement haché par rapport à des animaux comparables recevant de l'ensilage lacéré avec une machine à fléaux.

Nous rapporterons ici 4 essais, effectués de 1970 à 1973, dans lesquels nous avons comparé trois types de machine hachant ou lacérant plus ou moins finement le fourrage. Nous avons principalement étudié les effets sur les quantités ingérées par les animaux et sur leur croissance. Nous avons également fait varier le niveau d'apport d'aliment concentré aux animaux afin de savoir si l'influence de la finesse de hachage était modifiée ou non par ce niveau d'apport.

\section{MATÉRIËI, E'T MÉ'THODES}

Les 4 essais ont été réalisés, au domaine d'Orcival (Puy-de-Dôme) pour les 3 premiers, en I970-7I, I971-72 et I972-73, et au domaine de Marcenat (Cantal) pour le quatrième en I972-73. Dans chaque essai, nous avons préparé deux ensilages, l'un à brins courts, l'autre à brins moyens ou longs. Pour cela, trois machines ont été utilisées :

- une machine à tambour hacheur réglée pour hacher très finement (brins courts de 0,5 à $\mathrm{I}, 5 \mathrm{~cm}$ ),

- une machine à plateau hacheur hachant le fourrage en brins moyens de 5 à $\mathrm{I} 5 \mathrm{~cm}$,

- une machine à fléaux lacérant le fourrage en brins longs de Io à $25 \mathrm{~cm}$.

\section{Ensilages étudiés}

Pour chaque essai, nous avons rapporté dans le tableau I, l'espèce végétale, son stade de récolte, les machines de récolte utilisées, la dose d'acide formique cmployée et les incidents éventuels qui ont gêné la confection des ensilages. Dans chaque comparaison les deux ensilages n'ont pas été prépatés en même temps, mais successivement, le temps de remplissage de chaque silo étant 2 à 3 jours. Ils ont été tassés dans le silo au fur et à mesure du remplissage; dès la fin de celui-ci, ils ont été couverts d'un film plastique et chargés de paille. Les silos sont des "siloscouloirs " bétonnés de $100 \mathrm{~m}^{3}$ à Orcival et de $\mathrm{I} 50 \mathrm{~m}^{3}$ à Marcenat.

\section{Animaux}

Dans les 4 essais, nous avons utilisé des génisses de race laitière, Frisonne et Montbéliarde,

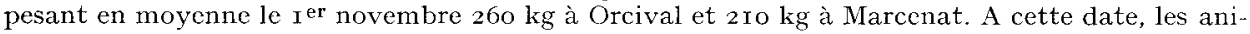
maux étaient âgés de Io mois à Orcival et de 8 mois à Marcenat. Ils ont été maintenus en stabulation entravée pendant toute la durée de l'expérience.

\section{Déroulement des essais (tabl. 2)}

Nos essais se sont déroulés de novembre à avril, selon un schéma à 2 périodes expérimentales avec inversion. Dans chaque essai les animaux ont été répartis en lots et appariés selon leur âge et leur poids, de 3 semaines à I mois après la rentrée à l'étable, alors qu'ils recevaient un régime 


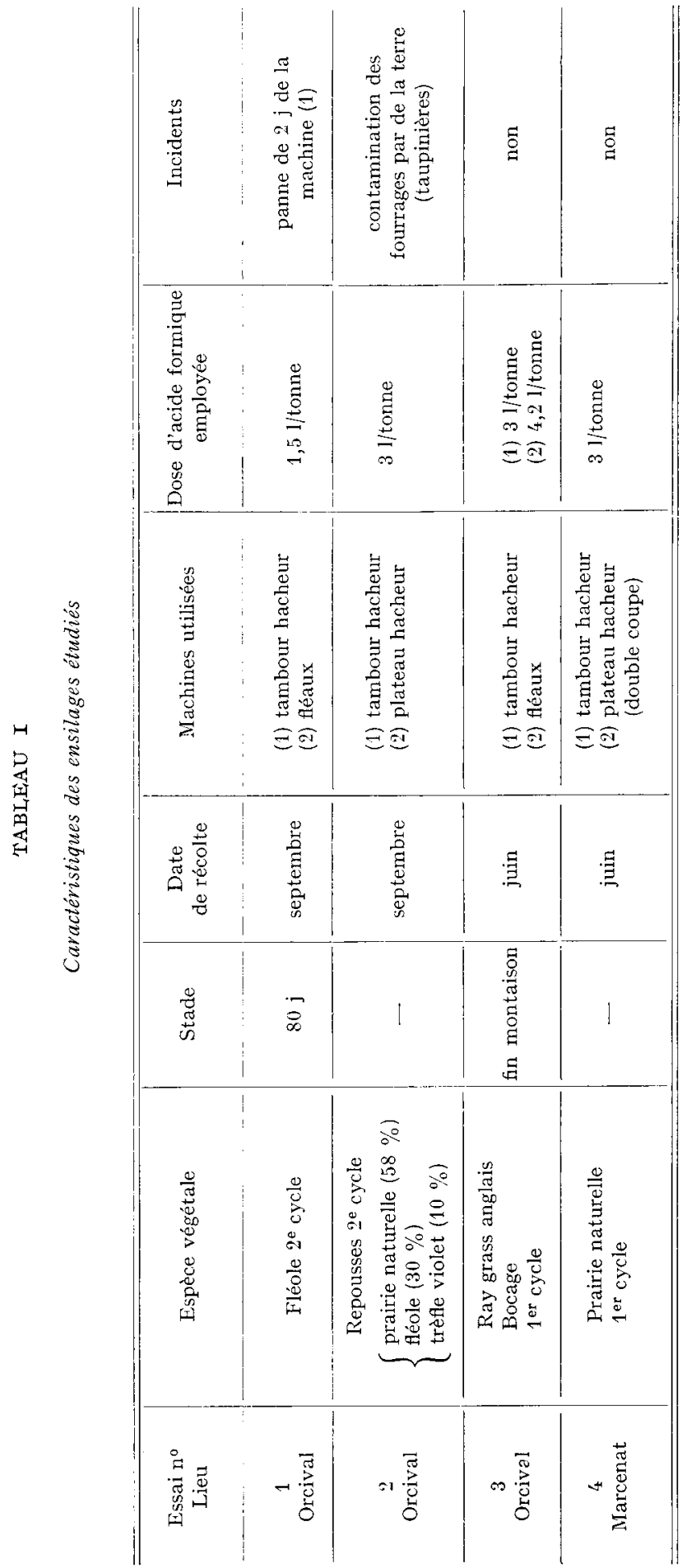




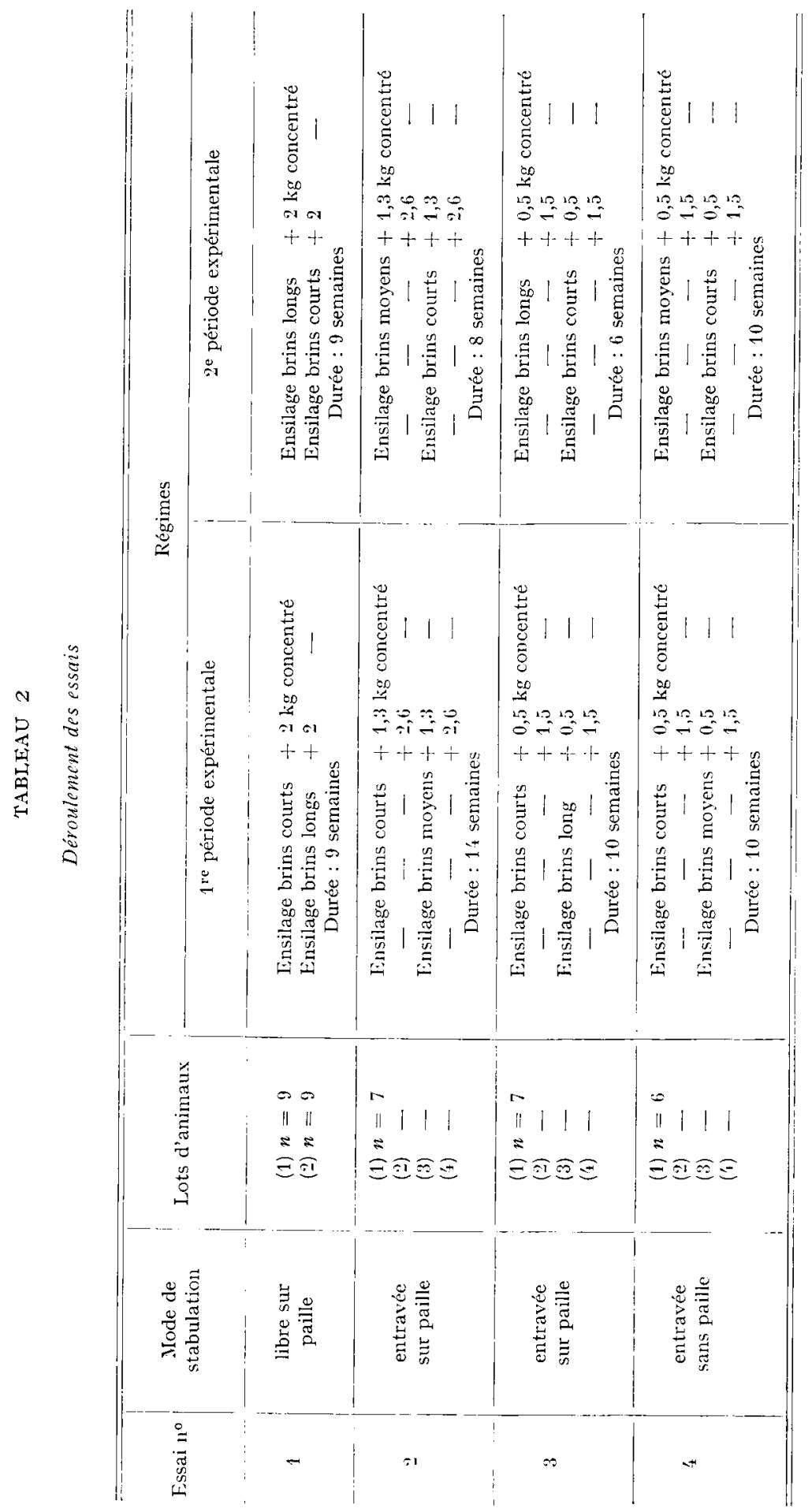


commun d'ensilage à volonté avec I $\mathrm{kg}$ d'aliment concentré (période pré-expérimentale). Puis les animaux ont reçu leurs régimes respectifs, les 2 premières semaines de chaque période expérimentale étant considérées comme période de mise en régime. Dans tous les essais les ensilages ont été distribués ad libitum ( Io p. Ioo de refus) en deux fois par jour (9 et i 7 hcures). Les animaux ont également reçu un aliment concentré comprenant i 5 p. Ioo d'orge, Io p, soo d'avoine, $20 \mathrm{p}$. Ioo de blé, 30 p. Ioo de maïs, I 2 p. I oo de tourtcau d'arachide, o,5 p. Ioo'de scl. Cet aliment concentré était apporté avec un mélange de sels minéraux le matin après l'enlèvoment des refus d'ensilage de lá veille et avant la distribution d'ensilage du matin.

\section{Mesures}

Les quantités ingérées par les génisscs ont été mesurées individuellement 4 jours par semaine (par pesées des quantités offertes et refusées), chaque semaine à Orcival et une scmaine sur deux à Marcenat. Les animaux ont été pesés durant deux jours consécutifs à la fin de la deuxième et de la dernière semaine des périodes expérimentales. Les gains de poids journaliers ont été calculés à partir de ces deux poids. A Orcival, nous avons mesuré la digestibilité des différents ensilages en utilisant un lot de 5 béliers castrés adultes de race Texel. Les béliers ont reçu l'ensilage ad libitum (Io p. Ioo de refus) en 2 repas par jour pendant 3 semaines; les mesures de quantités ingérées et les prélèvements de fèces ont été effectués lors de la $3^{\mathbf{e}}$ scmaine.

\section{Analyses}

La teneur en matière sèche des ensilages distribués à chaque repas et des refus journaliers a été détcrminée chaque jour de contrôle des quantités ingérées, par séchage à l'étuve à $80^{\circ} \mathrm{C}$. Elle a été corrigée pour les pertes de produits volatils lors du séchage par la formule suivante établie par Dulphy (résultats non publiés) à partir des produits volatils dosés avant ct après séchage sur 24 échantillons d'ensilages de graminées :

\section{MS corrigée $=\mathrm{Y} \times \mathrm{MS}$ non corrigée}

avec $\mathrm{Y}=\mathrm{I}, \mathrm{OO}+0,6 \mathrm{I} 6 x+0, \mathrm{I}+2 y+0,83 z+v$ ou $x, y$, z et $v$ représentent respectivement les quantités d'ammoniac, d'acide lactique, d'acides gras volatils et d'alcools dosés sur l'ensilage frais et exprimées en p. Ioo de la matière sèche non corrigée. Des échantillons d'ensilage ont été prélevés tout au long de chaque période expérimentale à des fin d'analyse plus complète. Ia teneur en matières azotées totales $(N$ Kjeldahl $\times 6,25)$ a été dosée sur ces échantillons frais. Sur les jus extraits par pression nous avons déterminé le pH, la teneur en ammoniac (CosWAY, 1957), en acide lactique (BARKER et SUMMERSON, I94I) et en acides gras volatils par chromatographie en phase gazeuse (RIGAud et JoURNET, 1970). Sur ces échantillons après séchage nous avons mesuré les teneurs en cellulose brute et en cendres. Les analyses des fèces de moutons (azote, cellulose brute de Weende, et cendres) ont été effectuées sur des échantillons secs

\section{RÉSULTATS}

Caractéristiques des ensilages utilisés (tabl. 3)

\section{Composition chimique.}

Comme nous l'avions observé précédemment (DULPHY et DEMarquiL, I, I973) il n'y a pas de différences importantes et systématiques dans les teneurs en cendres, en matières azotées et en cellulose brute entre les ensilages récoltés avec des machines différentes. Notons simplement que la quantité de terre dans les ensilages de l'essai 2 a été différente (différence notable de teneur en cendres) ; les deux machines utilisées étant munies d'un pick-up cette différence est peut-être due à l'hétérogénéité de la présence des taupinières dans la parcelle.

Dans l'essai I, l'ensilage haché finement a été mal conservé par suite d'une inter- 


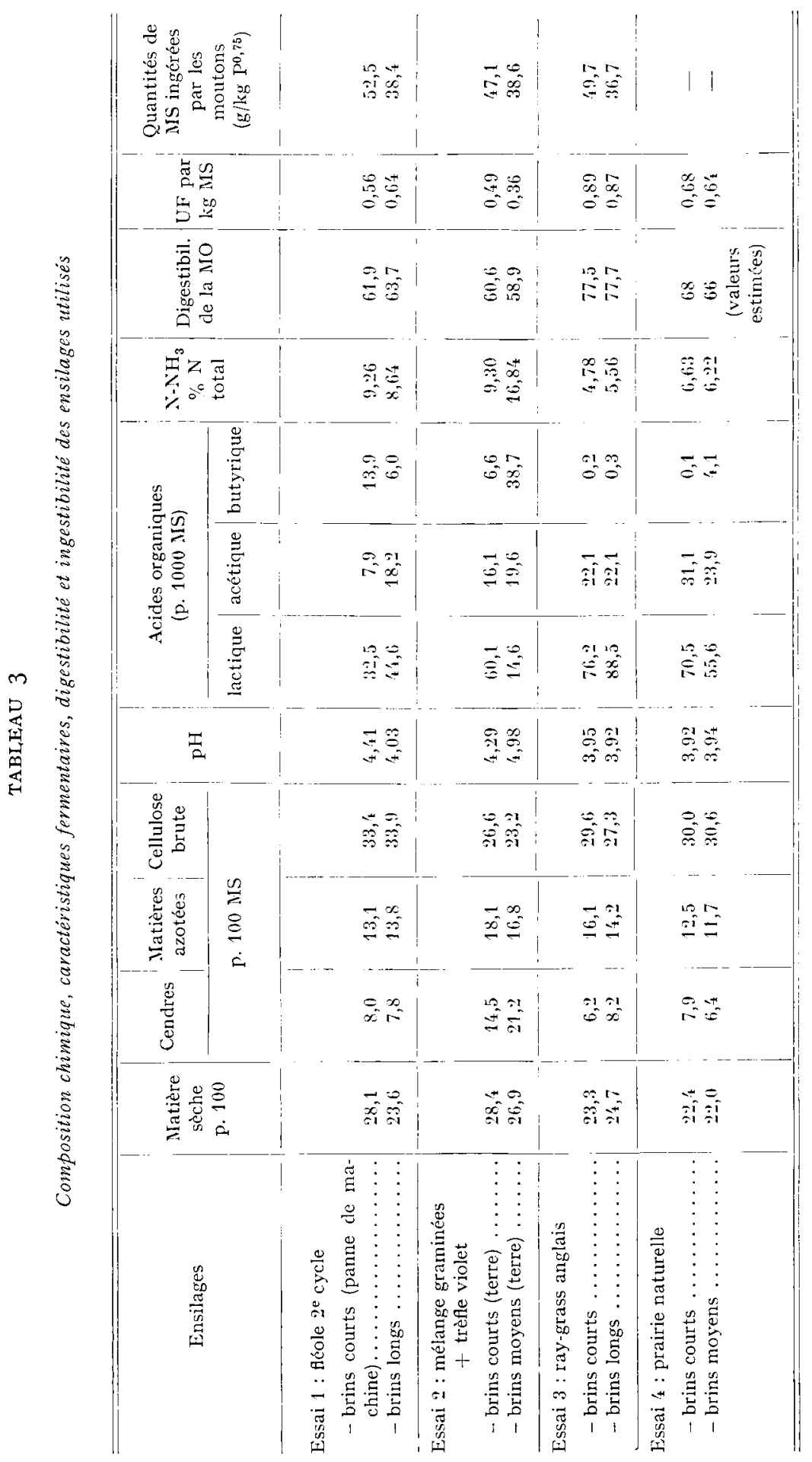


ruption du chantier de récolte causée par une panne de l'ensileuse à tambour-hacheur. Dans l'essai 2, les deux ensilages comparés (brins courts et brins moyens) ont été mal conservés par suite de la présence de nombreuses taupinières dans la parcelle récoltée.

Dans les deux derniers essais, les ensilages ont été très correctement conservés ct l'effet de la machine de récolte a été négligeable sur la qualité de conservation des ensilages.

En défnnitive, dans nos essais, 3 ensilages sur 8 ont été mal conservés, mais pour des causes diverses extérieures à l'expérimentation: panne de machine ou présence de taupinières dans les parcelles; les autres ensilages ont été conservés correctement. La faible influence des machines sur la qualité de conservation est vraisemblablement due à la présence d'acide formique comme conservateur (DUI,PHY et Demarguilly, I973).

Digestibilité et ingestibilité.

Compte tenu des incidents survenus lors de la confection des ensilages, il n'est pas possible d'imputer aux machines les différences de digestibilité enregistrées dans les essais I et 2 . Nous pouvons cependant rappeler que dans des essais précédents (I)Ulphy et DEMARQuiliy, I973), nous n'avions trouvé que des différences faibles et non significatives entre les digestibilités des ensilages récoltés avec différentes machines.

Dans les trois essais où elle a pu être mesurée, l'ingestibilité chez le mouton des trois ensilages à brins courts a été en moyenne de $49,8 \mathrm{~g} / \mathrm{kg} \mathrm{P} \mathrm{P}^{0,75}$ contre $37,9 \mathrm{~g}$ pour les ensilages à brins moyens ou longs correspondants. L'augmentation d'ingestibilité due au hachage fin a donc été de $+3 \mathrm{I}, 4 \mathrm{p}$. Ioo, valeur bien inférieure à celle que nous avions enregistrée lors des essais antérieurs $(+55.7 \mathrm{p}$. IOO, I)UL,pHy et 1)EMARQUII.IY, I973). Cette réponse plus faible s'explique peut-être, d'une part par le fait que le hachage fin ne s'est pas accompagné d'une amélioration de la qualité de la conservation ; d'autre part, parce que les ensilages ont été préparés à partir de fourrages pauvres en tiges (deux repousses et un premier cycle récolté à un stade précoce) avec lesquels les ensileuses à plateau hacheur ou à fléaux ont donné des brins plus courts que lors de; essais antérieurs effectués sur des fourrages de I $^{\text {rr }}$ cycle plus âgés.

\section{Quantités d'aliments ingérées par les génisses (tab1. 4)}

\section{Lffet de la machine de récolte.}

Dans les essais I et 3 , les quantités d'ensilage ingérées par les génisses ont été significativement $(\mathrm{P}<0,05)$ plus élevées (en moyenne de $6,4 \mathrm{p}$. Ioo) avec les ensilages à brins courts par rapport à ceux à brins longs, cela en présence de $\mathrm{I}, 5 \mathrm{~kg}$ d'aliment concentré en moyenne.

Dans les essais 2 et 4 , les quantités d'ensilage ingérées par les génisses n'ont augmenté que faiblement (en moyenne de $2,6 \mathrm{p}$. IOO) avec les ensilages à brins courts par rapport à ceux à brins moyens.

\section{Influence du niveau d'apport de l'aliment concentré.}

Dans les 3 essais dans lesquels nous avons fait varier le niveau d'apport de l'aliment concentré, nous avons enregistré, une diminution moyenne de $0,43 \mathrm{~kg}$ de matière sèche d'ensilage par kg de matière sèche d'aliment concentré apporté en sup- 
plément, et cela indépendamment de la finesse de hachage des ensilages. La diminution observée a été importante $(--0,72 \mathrm{~kg})$ dans l'essai 2 dans lequel les animaux recevaient une quantité élevée d'aliment concentré ( $2 \mathrm{~kg}$ en moyenne). Par contre, elle a été faible $(-0,28 \mathrm{~kg})$ dans les essais 3 et 4 dans lesquels les animaux ne recevaient en moyenne que I $\mathrm{kg}$ d'aliment concentré.

\section{TABIEAU 4}

Quantités de matiève sèche ingérées par les génisses et gains de poids journaliers

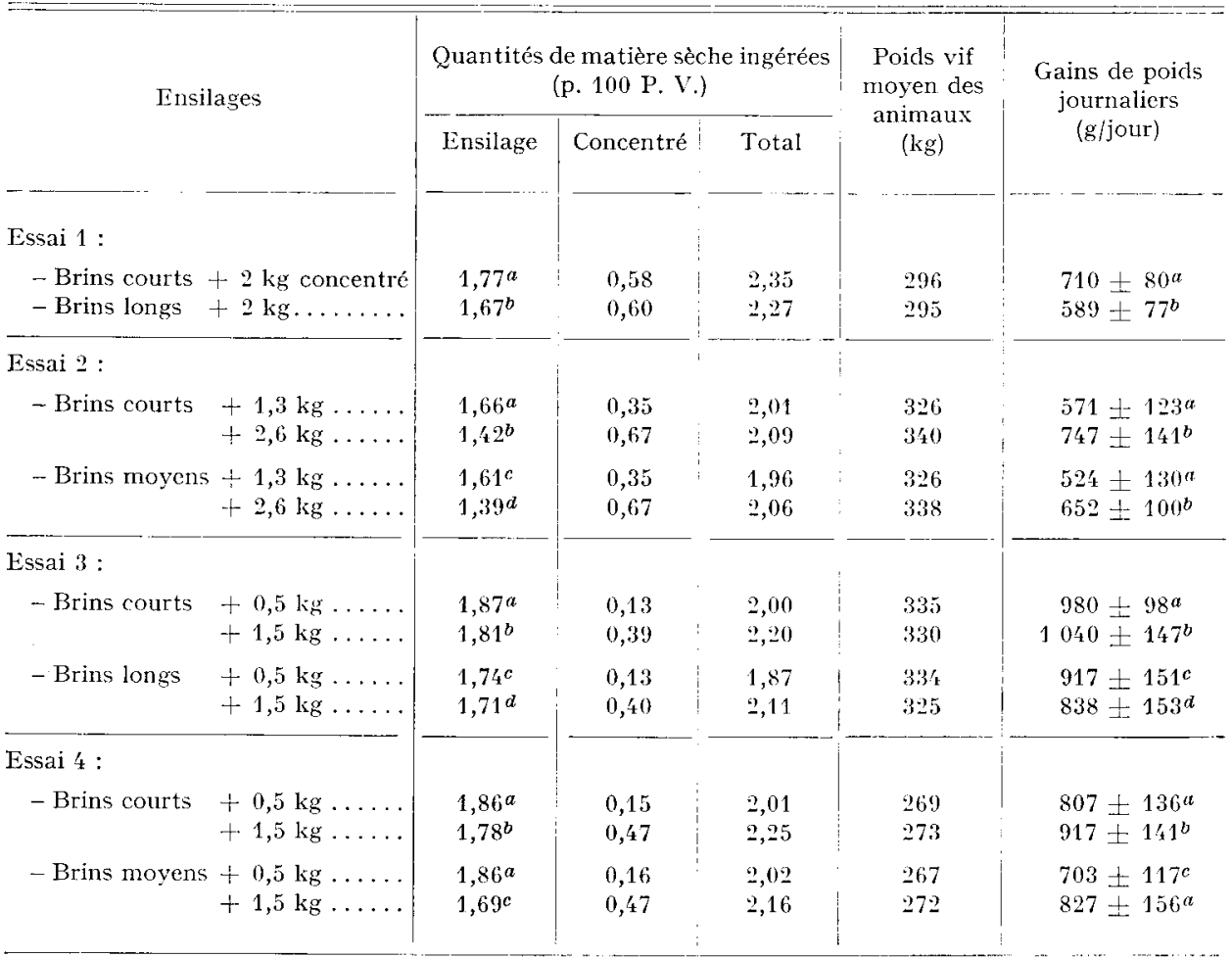

Dans un même essai, les valeurs portant en indice les mêmes lettres ne sont pas significativement différentes à la probabilité 0,05 .

\section{Croissance des génisses (tab1. 4)}

Effet de la méthode de récolte.

Dans les essais $\mathrm{I}$ et 3 , le gain de poids journalier des génisses a été significativement $(\mathrm{P}<0,05)$ plus élevé (en moyenne de $+\mathbf{I} 26 \mathrm{~g} /$ jour) avec l'ensilage à brins courts par rapport à l'ensilage à brins longs.

Dans les essais 2 et 4 , le gain de poids vif journalier des génisses a été plus élevé (en moyenne de $+84 \mathrm{~g} /$ jourr) avec l'ensilage à brins courts par rapport à l'ensilage à brins moyens. 
Il semble que l'effet de la finesse de hachage ait été plus important en distribuant une quantité élevée d'aliment concentré ( $+\mathrm{I} 27 \mathrm{~g} / \mathrm{jour}$ ) qu'une quantité faible $(+69 \mathrm{~g} /$ jour $)$, mais ces augmentations ne sont pas significativement différentes entre elles.

\section{Effet de la quantité d'aliment concentré.}

L'augmentation du gain de poids vif obtenu en apportant I kg brut d'aliment concentré supplémentaire dans les rations a été faible : en moyenne $+75 \mathrm{~g} /$ jour, (respectivement II7, - Io et II7 g/jour dans les essais 2, 3 et 4). Cependant, elle a été liée très étroitement au gain de poids des animaux ayant reçu le plus faible apport d'aliment concentré. En effet, si $Y$, est le gain de poids pour les animatrx ayant reçu I $\mathrm{kg}$ d'aliment concentré supplémentaire et $x$ le gain de poids journalier des animaux ayant reçu le plus faible apport d'aliment concentré, $\mathrm{Y}$ et $x$ sont liés de la manière suivante $: \mathrm{Y}=280+0,728 x\left(n=6, r=0,907^{*}\right)$ équation qui peut s'écrire : $\mathrm{Y}-x=280-0,272 x$.

La distribution d'un $\mathrm{kg}$ supplémentaire d'aliment concentré a donc entraîné une augmentation du gain de poids vif d'autant plus faible qu'en son absence le gain de poids vif des génisses était plus élevé : augmentation respective de $+\mathbf{I} 44$; +76 et $+8 \mathrm{~g} /$ jour suivant qu'en son absence le gain de poids vif était déjà de 500 , 750 et $\mathrm{I}$ ooo $\mathrm{g} / \mathrm{jour}$.

\section{Efficacité d'utilisation des rations}

On peut exprimer l'efficacité globale d'utilisation des rations (ensilage + aliment concentré) utilisées soit en $\mathrm{kg}$ de matière sèche totale ingérée par $\mathrm{kg}$ de gain de poids vif réalisé, soit en $\mathrm{kg}$ de matière organique digestible ingérée par $\mathrm{kg}$ de gain de poids vif. Pour ce dernier calcul nous avons pris comme digestibilité de la matière organique des ensilages, la digestibilité mesurée sur les moutons, (essais I à 3) soit estimée à partir de la composition chimique de l'ensilage (essai 4). Quant à la digestibilité de la matière organique de l'aliment concentré elle a été calculée à partir de la composition en céréales et tourteaux de cet aliment et de la digestibilité de ces céréales et tourteaux donnée par les tables de valeur nutritive des aliments.

\section{Effet de la finesse de hachage.}

Par kg de gain de poids vif, en présence d'une même quantité d'aliment concentré dans la ration (en moyenne $I, 22 \mathrm{~kg}$ de matière sèche), les génisses ont ingéré en moyenne $7,99 \mathrm{~kg}$ de matière sèche totale avec les rations d'ensilage à brins courts et $8,79 \mathrm{~kg}$ avec des rations d'ensilage à brins moyens ou longs. L'efficacité d'utilisation de la matière sèche des rations d'ensilages à brins courts a donc été supérieure de ro p. Ioo à celle des rations d'ensilages à brins moyens ou longs.

La matière organique digestible ingérée des rations d'ensilage à brins courts a de même été utilisée avec une meilleure efficacité $(+7,4$ p. Ioo) ; par $\mathrm{kg}$ de gain de poids les génisses ont ingéré $5,25 \mathrm{~kg}$ de matière organique digestible avec ces rations contre $5,64 \mathrm{~kg}$ avec les rations d'ensilages à brins moyens ou longs. En moyenne avec les rations d'ensilage à brins courts les génisses n'ont ingéré que $262 \mathrm{~g}$ de matière organique digestible $(+6,4 \mathrm{p}$. Ioo) de plus par jour qu'avec les rations d'ensilages à brins moyens ou longs mais ont eu un gain de poids nettement plus élevé, + $104 \mathrm{~g} /$ jour soit + I4,4 p. IoO. 


\section{Effet du niveau des apports d'aliment concentré.}

Dans les essais 2, 3, 4, où deux niveaux d'aliment concentré étaient distribués, un niveau " haut " et un niveau "bas " différant en moyenne de I, I kg (0,95 kg de matière sèche) par jour, les rations correspondant au niveau "haut " ont eu une efficacité d'utilisation très peu supérieure à celle des rations correspondant au niveau "bas ", respectivement $+\mathrm{I,9}$ et $+2,4$ p. Ioo pour la matière sèche et la matière organique digestible ingérée. Par $\mathrm{kg}$ de gain de poids vif, les génisses ont en effet ingéré 8,00 contre $8,15 \mathrm{~kg}$ de matière sèche totale et $5, \mathrm{Ig}$ contre $5,32 \mathrm{~kg}$ de matière organique digestible. En moyenne, avec les rations correspondant au " haut niveau" les génisses ont ingéré $559 \mathrm{~g}$ de matière organique digestible $(+\mathrm{I} 4,4 \mathrm{p}$. roo) de plus qu'avec les rations correspondant au «bas niveau " mais le gain de poids vif n'a été plus élevé que $87 \mathrm{~g}$ par jour ( + I I,6 p. IOO).

\section{DISCUSSION}

Contrairement à ce que nous avions trouvé antérieurement (DuLPHY et DEMARQUiLLY, I973) la machine de récolte n'a pas eu d'influence sur la qualité de conservation des ensilages. Les incidents divers de chantier (pannes de machine dans l'essai I, présence importante de taupinières dans l'essai 2) et l'emploi systématique de l'acide formique ont probablement masqué l'influence de la machine de récolte. D'autre part, ayant travaillé essentiellement sur des repousses, les différences de longueur de brins suivant le type de machine employé ont dû être atténuées. Les écarts d'ingestibilité mesurés sur moutons ont été plus faibles que dans nos essais précédents. Tout cela explique peut-être pourquoi le hachage fin n'a que peu augmenté la quantité d'ensilage ingérée par les génisses, augmentation maximum de 7,5 p. Ioo alors qu'elle a été de 27 , I à $3,5,5 \mathrm{p}$. roo dans d'autres essais (Dur,IHY et Michal,eT, en préparation). Quoi qu'il en soit il convient de souligner que l'augmentation de quantités ingérées avec la finesse de hachage des ensilages est beaucoup plus faible pour les génisses que pour les moutons. Ce phénomène est très intéressant ; les causes en sont actuellement à l'étude et seront discutées ultérieurement.

Malgré cela, le hachage fin a eu un effet positif très net sur le gain de poids vif journalier (augmentation moyenne de I04 g/jour) par comparaison à celui obtenu avec les ensilages à brins longs et moyens. Ces croits plus élevés résultent en partie de l'augmentation des quantités ingérées (en moyenne de $262 \mathrm{~g}$ de matière organique digestible par jour et par génisse) et en partie de l'amélioration de l'efficacité d'utilisation de la matière organique digestible ingérée. Ce deuxième effet est probable, le hachage fin ayant eu une action beaucoup plus nette que la distribution supplémentaire de I,I $\mathrm{kg}(0,95 \mathrm{~kg}$ de matière sèche) d'aliment concentré. L'apport supplémentaire de I,I kg d'aliment concentré a en effet entrainé un supplément de gain de poids de $87 \mathrm{~g} /$ jour/génisse en moyenne alors que les génisses ingéraient $559 \mathrm{~g}$ de matière organique digestible de plus par jour, du moins en supposant qu'il n'y a pas eu de digestibilité associative entre le fourrage et le concentré. Cependant, il est possible que l'apport supplémentaire d'aliment concentré (jusqu'à $30 \mathrm{p}$. Ioo de la ration totale) ait entraîné une baisse de la digestibilité du fourrage et 
par là de la quantité de matière organique digestible ingérée. Quoi qu'il en soit tout se passe dans ces essais comme si l'augmentation de la quantité d'énergie ingérée se traduit surtout par une modification de la composition du croit (vraisemblablement par suite d'une modification des produits terminaux de la digestion) quand le supplément d'énergie ingéré est apporté sous forme d'aliment concentré, alors qu'elle se traduit essentiellement par une augmentation de croissance quand ce supplément d'énergie résulte de l'augmentation des quantités d'ensilage ingérées par suite du hachage fin. I1 est d'ailleurs curieux de constater que l'augmentation des croîts journaliers consécutifs au hachage fin n'a pas été liée au niveau des apports d'aliment concentré et qu'elle a même eu tendance a être un peu plus élevée avec le niveau haut qu'avec le niveau bas (respectivement +127 et $+69 \mathrm{~g} /$ jour).

Quoi qu'il en soit, il est vraisemblable que l'effet du hachage fin sur le gain de poids vif journalier aurait été beaucoup plus élevé si comme dans nos autres essais sur génisses (Dulphy et Mrchaler en préparation) ou sur vaches (Dulphy et DEMARQUil.LY, I975) l'augmentation de quantité ingérée avait été plus importante.

D'un point de vue zootechnique ces essais montrent qu'il est possible d'obtenir des gains de poids journaliers de 600 à $800 \mathrm{~g}$ en distribuant à des génisses de I an un ensilage de graminées comme seul aliment à condition de récolter le fourrage à un stade précoce, avec une machine hachant finement et de bien le conserver. Ce sont d'ailleurs les croissances obtenues par WAL.DO (I973) avec des fourrages à base de luzerne-dactyle, hachés finement, ensilés avec addition d'acide formique et distribués à volonté, sans aliment concentré à des génisses Holstein de I an. Si ces conditions ne sont pas remplies, il faudra apporter en supplément de l'ensilage I à $2 \mathrm{~kg}$ d'aliment concentré, voire même $3 \mathrm{~kg}$ si l'ensilage est récolté tardivement et mal conservé

$$
\text { Regit pour publication en mars } 1975 .
$$

\section{SUMMARY}

INFLUENCE OF THE TYPE OF FORAGE HARVESTER

ON THE VAIUE OF GRASS SIIAGES FOR HEIFERS OF DAIRY BREEDS

I. Dairy heifers were used to compare the feeding value of silage grasses harvested with three different machines: precision chop forage harvester (finely chopped silage), harvester with knives on a plate (meanly chopped silage), flail harvester (coarsely chopped silage). In 2 trials, the precision chop harvester was compared with the harvester giving meanly chopped silage and in 2 other trials it was compared with the flail harvester (table $\mathbf{I}$ ). Formic acid was added to all forages at harvesting and the type of harvester only slightly affected the preservation quality of the silages (table 3 ).

2. In each trial, silages were offered ad libitum without hay to comparable groups of I year old heifers. In addition, the animals received a concentrate-feed, in equal amounts in trial $\mathbf{I}$ and according to 2 feeding levels in trials 2 to 4 (each group being divided into 2 sub-groups) (table 2 ). All experiments were carried out in winter according to a plan comprising 2 experimental periods with inversion of the silage in the middle of the winter

3. The average silage intake of the heifers was significantly higher $(6.4 \mathrm{p}$. I00, $\mathrm{P}<0.05)$ with finely chopped silages as compared to the coarsely chopped ones. On an average, it only increased a little (2.6 p. Ioo) with the finely chopped silages as compared to the meanly chopped ones (table 4 ).

When varying the concentrate feed level, a mean decrease of $0.43 \mathrm{~kg}$ silage dry matter per $\mathrm{kg}$ dry matter of the concentrate feed supplement was recorded independently of the chopping 
fineness of the silages. The daily liveweight gain of the heifers was $+126 \mathrm{~g} /$ day higher with the finely chopped silage as compared to the coarsely chopped one, and $84 \mathrm{~g} /$ day higher with the finely chopped silage as compared to the meanly chopped one. The effect of chopping fineness was more marked when offering a high amount of concentrate feed ( + I $27 \mathrm{~g} / \mathrm{day}$ ) than when offering a low amount $(+69 \mathrm{~g} / \mathrm{day})$, but these increases were not significantly different the one from the others.

The increase in liveweight gain obtained by supplementing the diets with $\mathrm{I}$ kg concentrate feed was small (on an average $75 \mathrm{~g} /$ day). It was all the smaller as the weight gain of the animals having received the lowest concentrate supply was high.

4. The higher performances of the heifers with finely chopped silages seem partly to result from the increase in the intake of digestible organic matter and mainly from the improvement of the utilization efficiency of this digestible organic matter.

On the other hand, the increase in the intake level of digestible organic matter resulting from the rise in the amount of concentrate feed offered seems principally to lead to a modification of the composition of the gain. All these results are discussed.

\section{RÉFÉRENCES BIBLIOGRAPHIQUE'S}

Barker J. B., Summerson W. H., i94I. The colorimetric determination of lactic acid in biological material. J. Biol. Chem., 138, 535-554.

Conway E. J., 1957. Microdiffusion analysis and volumetric error. Crosby, Lockwood. London.

Dulphy J. P., Demarquiliy C., i973. Influence de la machine de récolte et de la finesse de hachage sur la valeur alimentaire des ensilages. Ann. Zootech, 22, r99-2I7.

Dulphy J.-P., Demarouilly $\quad$ C., I975. Influence de la machine de récolte sur les quantités d'ensilage ingérées et les performances des vaches laitieres. Ann. Zoolech., 24, 363-37r.

Rigaud J., Journet M., ig7o. Méthode de dosage des acides gras volatils dans le liquide du rumen. Ann. Biol. anim. Bioch. Biophys., 10, I5 I- 57 .

Roche J. F., O'ShEA J., FiYx A. V., I972. liffect of stocking density and length of chop on performance of heifer at a self feeding silage face. An foras Taluntais Research Report Animal Production, I8-19.

Waldo D. R., r973. Chenical pieservation of forages. Proc. Comell Nutr. Conf. Feed Manufact., $50-58$ 